\title{
Dietary sodium citrate improved oxidative stability in red hybrid tilapia (Oreochromis sp.) but reduced growth, health status, intestinal short chain fatty acids and induced liver damage
}

\begin{abstract}
Among organic acids, citric acid and their salts are currently the most studied as a supplement in aquafeeds to potentially improve growth and nutrient utilization in fish. The results have been generally beneficial but no studies have been performed on tilapia. A 50 day experiment was conducted on the effects of dietary sodium citrate at $0,1,2$ and $4 \%$ on the growth, feeding efficiency, body indices, muscle proximate composition, muscle lipid peroxidation, some plasma and blood parameters, intestinal short chain fatty acids (SCFA), and liver histopathology of red hybrid tilapia (Oreochromis sp.). Triplicate groups of 60 tilapia fingerlings (initial weight of $1.86 \pm 0.01$ ) were in each treatment. Results showed that, while not significant, increasing dietary sodium citrate reduced tilapia growth $(\mathrm{p}>0.05)$. However, muscle crude protein $(\mathrm{r} 2=0.931)$, lipid $(\mathrm{r} 2=0.962)$, and ash $(\mathrm{r} 2=0.834)$ significantly decreased at increasing dietary sodium citrate levels $(\mathrm{p}<0.05)$. Plasma ALT significantly increased $(\mathrm{p}<0.05 ; \mathrm{r} 2=0.357)$ with increasing dietary sodium citrate treatments along with histopathological liver damage that included hemorrhages, necrosis and inflammatory responses. Many of the cell differential counts were significantly ( $p<0.05$ ) altered by increasing dietary sodium citrate levels. Among the intestinal SCFA in tilapia, acetic acid was the highest, followed by propionic and butyric acid, and these all significantly decreased $(\mathrm{p}<0.05)$ with increasing dietary sodium citrate. In all dietary sodium citrate treatments, muscle lipid peroxidation was significantly less $(\mathrm{p}<0.05 ; \mathrm{r} 2=0.211)$ indicating increased oxidative stability. While dietary sodium citrate was toxic to tilapia at the levels used, and is not recommended as a supplement, the decreased lipid peroxidation warrants further investigation with other species. Such research may have important implications for filet quality over prolonged storage. Statement of relevance: Sodium citrate reduced growth but may increase shelf-life.
\end{abstract}

Keyword: Organic acid; Oxidative stability; Histology; Short chain fatty acids; Liver damage; Phagocytic activity; Phytic acid 